erhebliche Zweifel, ob sich durch den Einnahmezeitpunkt während einer chronischen Verordnung tatsächlich „die Metamizol-ASS-Interaktion praktisch immer zuverlässig“ vermeiden lässt.

\section{Korrespondenzadresse}

Priv.-Doz. Dr. H. Bornemann-Cimenti Universitätsklinik für Anästhesiologie und Intensivmedizin, Medizinische Universität Graz Auengriggerplatz 29, 8036 Graz, Deutschland helmar.bornemann@medunigraz.at
Interessenkonflikt. H. Bornemann-Cimenti, S. Fleck und G. Rumpold Seitlinger geben an, dass kein Interessenkonflikt besteht.

\section{Literatur}

1. Achilles A, Mohring A, Dannenberg L et al (2017) Analgesic medication with dipyrone in patients with coronary artery disease: Relation to MACCE. Int J Cardiol 236:76-81

2. Dannenberg L, Erschoff V, Bonner F et al (2016) Dipyrone comedication in aspirin treated stroke patients impairs outcome. Vascul Pharmacol 87:66-69

3. Dingermann T (2017) Metamizol: Something to think about. Z Rheumatol 76(5):377. doi:10.1007/ s00393-017-0317-1
4. Levy M, Zylber-KatzE, Rosenkranz B (1995) Clinica pharmacokinetics of dipyrone and its metabolites. Clin Pharmacokinet 28:216-234

5. Polzin A, Richter S, Schror Ket al (2015) Prevention of dipyrone (metamizole) induced inhibition of aspirin antiplatelet effects. Thromb Haemost 114:87-95

6. Schmitz A, Romann L, Kienbaum P et al (2017) Dipyrone (metamizole) markedly interferes with platelet inhibition by aspirin in patients with acute and chronic pain: a case-control study. Eur J Anaesthesiol 34:288-296
Z Rheumatol 2017 · 76:553

DOI 10.1007/s00393-017-0336-y

C) Springer Medizin Verlag GmbH 2017

CrossMark

\section{T. Dingermann}

Institut für Pharmazeutische Biologie, Biozentrum, Goethe-Universität Frankfurt, Frankfurt, Deutschland

\title{
Eine wichtige Ergänzung zu meinem Beitrag
}

\section{Erwiderung}

Zum Leserbrief von Priv.-Doz. Dr. Helmar Bornemann-Cimenti, Dr. Sabine Fleck, Ass.Prof. Dr. Gudrun Rumpold Seitlinger. Ist die Verschiebung des Applikationszeitpunkts zur Vermeidung einer Aspirin/MetamizolInteraktion bei chronischer Gabe ausreichend? doi: 10.1007/s00393-017-0332-2.

Originalbeitrag: Dingermann T (2017) Metamizol: Something to think about. Z Rheumatol doi: 10.1007/s00393-017-03171.

Die Zuschrift von Bornemann-Cimenti et al. sehe ich in keiner Weise als Widerspruch, sondern als eine wichtige Ergän- zung zu meinem Beitrag. Sie unterstreicht noch deutlicher das Interaktionsproblem und fordert unbedingte Beachtung ein.

\section{Korrespondenzadresse}

\section{Prof. Dr. T. Dingermann}

Institut für Pharmazeutische Biologie, Biozentrum, Goethe-Universität Frankfurt Max-von-Laue-Str. 9, 60438 Frankfurt, Deutschland dingermann@em.uni-frankfurt.de

Interessenkonflikt. T. Dingermann gibt an, dass kein Interessenkonflikt besteht. 